\title{
Chaos in quantum steering in high-dimensional systems
}

\author{
Guang Ping $\mathrm{H} \oplus^{*}$ \\ School of Physics, Sun Yat-sen University, Guangzhou 510275, China
}

\begin{abstract}
Quantum steering means that in some bipartite quantum systems the local measurements on one side can determine the state of the other side. Here we show that in high-dimensional systems there exists a specific entangled state which can display a kind of chaos effect when being adopted for steering. That is, a subtle difference in the measurement results on one side can steer the other side into completely orthogonal states. Moreover, by expanding the result to infinite-dimensional systems, we find two sets of states for which, contrary to common belief, even though their density matrices approach being identical, the steering between them is impossible. This property makes them very useful for quantum cryptography.
\end{abstract}

PACS numbers: 03.65.Ud, 03.67.Mn, 03.67.Bg, 03.65.Ta

\section{INTRODUCTION}

The concept of quantum steering (a.k.a., entanglement steering or Einstein-Podolsky-Rosen steering), first introduced by Schrödinger in 1935 [1, 2], is a generalization of the "spooky action at a distance" proposed by Einstein, Podolsky and Rosen [3]. It means that some bipartite quantum systems can display a kind of nonlocality, such that the local measurements on one side of the system can affect (i.e., "steer") the state of the other side. Recently, with technological advances in handling quantum entangled states, the theoretical researches on quantum steering became even more active [4 10], with research topics ranging from the relationship between steering, entanglement, and nonlocality to the quantifying of steering, and the criterion of steerability (i.e., whether steering can be observed in a specific quantum state), etc.

Here we are interested in two questions.

(i) Given a steerable bipartite system $\alpha \otimes \beta$ such that two local measurements on $\alpha$ can steer $\beta$ into two highly distinguishable states, respectively, will the two measurements always be highly distinguishable too?

(ii) Given two sets of states which have identical density matrices, is it always possible to find a bipartite system that could steer the state of its subsystem between the elements of the two sets?

In the next section, we will answer the first question by showing that there exists a specific entangled state in high-dimensional systems, which can display a kind of chaos effect in steering, i.e., a very subtle difference in the measurement results on $\alpha$ can steer $\beta$ into completely orthogonal states. Then in Sec. III, we will answer the second question by proposing two sets of states in infinite-dimensional systems whose density matrices approach each other, and show that contrary to common belief, it is impossible to find a bipartite system that can realize the steering between these states. The significance of this result will be elaborated in Sec. IV.

\section{CHAOS EFFECT IN FINITE-DIMENSIONAL SYSTEMS}

Consider a bipartite system $\alpha \otimes \beta$ prepared in the entangled state

$$
|\Omega\rangle=\frac{1}{\sqrt{n-1}} \sum_{i=1}^{n-1}\left|\alpha_{i+}\right\rangle_{\alpha}\left|\phi_{i+}\right\rangle_{\beta} .
$$

Here $\alpha$ and $\beta$ are both $n$-dimensional systems, with $\left|\alpha_{i+}\right\rangle_{\alpha}$ and $|i\rangle_{\beta}(i=0, \ldots, n-1)$ being their orthonormal bases, respectively (the subscripts $\alpha$ and $\beta$ will be omitted thereafter), and

$$
\left|\phi_{i+}\right\rangle \equiv \frac{1}{\sqrt{2}}(|0\rangle+|i\rangle)
$$

*Electronic address: hegp@mail.sysu.edu.cn 
for $i=1, \ldots, n-1$. Now we will show that Eq. (1) can display a kind of chaos effect when we try to steer the state of $\beta$ by measuring system $\alpha$.

Defining

$$
\left|\phi_{i-}\right\rangle \equiv \frac{1}{\sqrt{2}}(|0\rangle-|i\rangle)
$$

for $i=1, \ldots, n-1$, and

$$
\left|\phi_{n \pm}\right\rangle \equiv \frac{1}{\sqrt{n}}\left(|0\rangle \mp \sum_{i=1}^{n-1}|i\rangle\right)
$$

we can see that the sets $B_{ \pm} \equiv\left\{\left|\phi_{i \pm}\right\rangle, i=1, \ldots, n\right\}$ form two complete (but nonorthogonal) bases of the $n$-dimensional system $\beta$.

Expanding each $\left|\phi_{i+}\right\rangle(i=1, \ldots, n-1)$ in the basis $B_{-}$, we have

$$
\left|\phi_{i+}\right\rangle=\frac{2-n}{n}\left|\phi_{i-}\right\rangle+\sum_{i^{\prime}=1, i^{\prime} \neq i}^{n-1} \frac{2}{n}\left|\phi_{i^{\prime}-}\right\rangle+\sqrt{\frac{2}{n}}\left|\phi_{n-}\right\rangle .
$$

Substituting it into Eq. (11) gives

$$
\begin{aligned}
|\Omega\rangle= & \frac{1}{\sqrt{n-1}}\left(\sum_{i=1}^{n-1} \frac{2-n}{n}\left|\alpha_{i+}\right\rangle\left|\phi_{i-}\right\rangle+\sum_{i=1}^{n-1} \sum_{i^{\prime}=1, i^{\prime} \neq i}^{n-1} \frac{2}{n}\left|\alpha_{i+}\right\rangle\left|\phi_{i^{\prime}-}\right\rangle\right) \\
& +\sqrt{\frac{2}{n}}\left(\frac{1}{\sqrt{n-1}} \sum_{i=1}^{n-1}\left|\alpha_{i+}\right\rangle\right)\left|\phi_{n-}\right\rangle .
\end{aligned}
$$

Rearranging the second term on the right yields

$$
\begin{aligned}
|\Omega\rangle= & \frac{1}{\sqrt{n-1}} \sum_{i=1}^{n-1}\left(\frac{2-n}{n}\left|\alpha_{i+}\right\rangle+\frac{2}{n} \sum_{i^{\prime}=1, i^{\prime} \neq i}^{n-1}\left|\alpha_{i^{\prime}+}\right\rangle\right)\left|\phi_{i-}\right\rangle \\
& +\sqrt{\frac{2}{n}}\left(\frac{1}{\sqrt{n-1}} \sum_{i=1}^{n-1}\left|\alpha_{i+}\right\rangle\right)\left|\phi_{n-}\right\rangle .
\end{aligned}
$$

Define

$$
\left|\tilde{\alpha}_{i-}\right\rangle \equiv c_{-}\left(\frac{2-n}{n}\left|\alpha_{i+}\right\rangle+\frac{2}{n} \sum_{i^{\prime}=1, i^{\prime} \neq i}^{n-1}\left|\alpha_{i^{\prime}+}\right\rangle\right)
$$

for $i=1, \ldots, n-1$, where the normalization constant

$$
c_{-} \equiv \frac{1}{\sqrt{\left(\frac{2-n}{n}\right)^{2}+(n-2)\left(\frac{2}{n}\right)^{2}}}=\frac{1}{\sqrt{1-4 / n^{2}}} .
$$

Also define

$$
\left|\tilde{\alpha}_{n-}\right\rangle \equiv \frac{1}{\sqrt{n-1}} \sum_{i=1}^{n-1}\left|\alpha_{i+}\right\rangle
$$

then Eq. (7) becomes

$$
|\Omega\rangle=\frac{1}{\sqrt{n-1}} \sum_{i=1}^{n-1} \frac{1}{c_{-}}\left|\tilde{\alpha}_{i-}\right\rangle\left|\phi_{i-}\right\rangle+\sqrt{\frac{2}{n}}\left|\tilde{\alpha}_{n-}\right\rangle\left|\phi_{n-}\right\rangle .
$$


For a given $i(i \in\{1, \ldots, n-1\})$, if we apply the measurement $\left\{\left|\tilde{\alpha}_{i-}\right\rangle\left\langle\tilde{\alpha}_{i-}|, I-| \tilde{\alpha}_{i-}\right\rangle\left\langle\tilde{\alpha}_{i-}\right|\right\}$ (where $I$ is the identity operator) on system $\alpha$, there is a small but non-vanishing probability that the projection $\left|\tilde{\alpha}_{i-}\right\rangle\left\langle\tilde{\alpha}_{i-}\right|$ will be successful. In this case, Eq. (11) shows that system $\beta$ will collapse to

$$
\begin{aligned}
\left|\tilde{\phi}_{i-}\right\rangle \equiv & c^{\prime}\left(\frac{1}{c_{-} \sqrt{n-1}}\left(\left|\phi_{i-}\right\rangle+\sum_{i^{\prime}=1, i^{\prime} \neq i}^{n-1}\left\langle\tilde{\alpha}_{i-} \mid \tilde{\alpha}_{i^{\prime}-}\right\rangle\left|\phi_{i^{\prime}-}\right\rangle\right)\right. \\
& \left.+\sqrt{\frac{2}{n}}\left\langle\tilde{\alpha}_{i-} \mid \tilde{\alpha}_{n-}\right\rangle\left|\phi_{n-}\right\rangle\right)
\end{aligned}
$$

where the normalization constant

$$
c^{\prime}=\sqrt{\frac{n(n-1)(n+2)}{\left(n^{2}+2\right)}} .
$$

From Eqs. (8) and (10) we can derive

$$
\left\langle\tilde{\alpha}_{i-} \mid \tilde{\alpha}_{i^{\prime}-}\right\rangle=\frac{-4}{n^{2}-4}
$$

for any $i \neq i^{\prime}\left(i, i^{\prime} \in\{1, \ldots, n-1\}\right)$, and

$$
\left\langle\tilde{\alpha}_{i-} \mid \tilde{\alpha}_{n-}\right\rangle=\frac{\sqrt{n-2}}{\sqrt{n-1} \sqrt{n+2}}
$$

for any $i(i \in\{1, \ldots, n-1\})$. Substituting them and $c_{-}=1 / \sqrt{1-4 / n^{2}}$ into Eq. (12), we have

$$
\begin{aligned}
\left|\tilde{\phi}_{i-}\right\rangle= & c^{\prime}\left(\frac{\sqrt{1-4 / n^{2}}}{\sqrt{n-1}}\left(\left|\phi_{i-}\right\rangle-\frac{4}{n^{2}-4} \sum_{i^{\prime}=1, i^{\prime} \neq i}^{n-1}\left|\phi_{i^{\prime}-}\right\rangle\right)\right. \\
& \left.+\frac{\sqrt{2 / n} \sqrt{n-2}}{\sqrt{n-1} \sqrt{n+2}}\left|\phi_{n-}\right\rangle\right) .
\end{aligned}
$$

Thus we obtain Result 1.

Result 1: If we manage to project system $\alpha$ into the state $\left|\tilde{\alpha}_{i-}\right\rangle$ defined in Eq. (8), then $\beta$ will collapse into the state $\left|\tilde{\phi}_{i-}\right\rangle$ in Eq. (16).

On the other hand, from Eq. (11) we can see that for the same given $i$, if we apply the measurement $\left\{\left|\alpha_{i+}\right\rangle\left\langle\alpha_{i+}\right|, I-\right.$ $\left.\left|\alpha_{i+}\right\rangle\left\langle\alpha_{i+}\right|\right\}$ on system $\alpha$ instead, there is also a small but non-vanishing probability that the projection $\left|\alpha_{i+}\right\rangle\left\langle\alpha_{i+}\right|$ will be successful. In this case, the resultant state of system $\beta$ can be obtained directly from Eq. (1), yielding Result 2 .

Result 2: If we manage to project system $\alpha$ into the state $\left|\alpha_{i+}\right\rangle$, then $\beta$ will collapse into the state $\left|\phi_{i+}\right\rangle$ in Eq. (2).

Now let us study the relationship between the states in Results 1 and 2. From Eq. (8) we find

$$
\left|\left\langle\alpha_{i+} \mid \tilde{\alpha}_{i-}\right\rangle\right|^{2}=\left|\frac{2-n}{n} c_{-}\right|^{2}=1-\frac{4}{n+2},
$$

i.e., $\left|\alpha_{i+}\right\rangle$ and $\left|\tilde{\alpha}_{i-}\right\rangle$ are very close to each other when $n$ is high. In contrast, multiplying $\left\langle\phi_{i+}\right|$ by the right-hand side 
of Eq. (16), we have

$$
\begin{aligned}
\left\langle\phi_{i+} \mid \tilde{\phi}_{i-}\right\rangle= & c^{\prime}\left(\frac{\sqrt{1-4 / n^{2}}}{\sqrt{n-1}}\left(\left\langle\phi_{i+} \mid \phi_{i-}\right\rangle-\frac{4}{n^{2}-4} \sum_{i^{\prime}=1, i^{\prime} \neq i}^{n-1}\left\langle\phi_{i+} \mid \phi_{i^{\prime}-}\right\rangle\right)\right. \\
& \left.+\frac{\sqrt{2 / n} \sqrt{n-2}}{\sqrt{n-1} \sqrt{n+2}}\left\langle\phi_{i+} \mid \phi_{n-}\right\rangle\right) \\
= & c^{\prime}\left(\frac{\sqrt{1-4 / n^{2}}}{\sqrt{n-1}}\left(0-\frac{4}{n^{2}-4}(n-2) \frac{1}{2}\right)\right. \\
& \left.+\frac{\sqrt{2 / n} \sqrt{n-2}}{\sqrt{n-1} \sqrt{n+2}} \sqrt{2 / n}\right) \\
= & 0
\end{aligned}
$$

for any $n$, i.e., $\left|\phi_{i+}\right\rangle$ and $\left|\tilde{\phi}_{i-}\right\rangle$ are always strictly orthogonal to each other. Recall that Results 1 and 2 mean that projecting system $\alpha$ into $\left|\alpha_{i+}\right\rangle$ (or $\left|\tilde{\alpha}_{i-}\right\rangle$ ) will make system $\beta$ collapse into $\left|\phi_{i+}\right\rangle$ (or $\left|\tilde{\phi}_{i-}\right\rangle$ ). Then with the fact that $\left|\alpha_{i+}\right\rangle$ and $\left|\tilde{\alpha}_{i-}\right\rangle$ are very close while $\left|\phi_{i+}\right\rangle$ and $\left|\tilde{\phi}_{i-}\right\rangle$ are strictly orthogonal, we obtain Conclusion 1.

Conclusion 1: There exists a certain form of states [see, e.g., Eq. (1)] in high-dimensional systems, such that when it is adopted for quantum steering, we can observe the following "chaos" behavior: a very subtle difference on the measurement results on $\alpha$ can lead to completely orthogonal steering results on $\beta$.

However, at first glance this chaos effect seems hard to find because, as stated above, for a given $i$ if we apply the measurement $\left\{\left|\tilde{\alpha}_{i-}\right\rangle\left\langle\tilde{\alpha}_{i-}|, I-| \tilde{\alpha}_{i-}\right\rangle\left\langle\tilde{\alpha}_{i-}\right|\right\}$ on system $\alpha$, the projection $\left|\tilde{\alpha}_{i-}\right\rangle\left\langle\tilde{\alpha}_{i-}\right|$ will be successful with a very small probability only. Meanwhile, for any finite $n$, Eqs. (8) and (10) show that $\left\{\left|\tilde{\alpha}_{i-}\right\rangle, i=1, \ldots, n\right\}$ is a nonorthogonal set, so that we cannot use it as an orthogonal measurement basis. Nevertheless, $\left\{\left|\alpha_{i+}\right\rangle, i=0,1, \ldots, n-1\right\}$ is an orthogonal measurement basis, as this is how it was defined below Eq. (1). Suppose that we prepare the state $|\Omega\rangle$ in Eq. (1) and apply the complete measurement $\left\{\left|\alpha_{i+}\right\rangle\left\langle\alpha_{i+}\right|, i=0,1, \ldots, n-1\right\}$ on $\alpha$. In this case the measurement device will surely produce an output $i_{0} \in\{1, \ldots, n-1\}$. Then from Result 2 , we will assume that $\alpha$ was projected to $\left|\alpha_{i_{0}}\right\rangle$ successfully and system $\beta$ was steered into $\left|\phi_{i_{0}+}\right\rangle$. But in practice, there could be a chance that, due to the imprecision of the measurement device, $\alpha$ may actually be projected into $\left|\tilde{\alpha}_{i_{0}-}\right\rangle$ since Eq. (17) indicates that it is very close to $\left|\alpha_{i_{0}+}\right\rangle$ when $n$ is high. Theoretically, this is equivalent to applying the measurements $\left\{\left|\tilde{\alpha}_{i_{0}-}\right\rangle\left\langle\tilde{\alpha}_{i_{0}-}|, I-| \tilde{\alpha}_{i_{0}-}\right\rangle\left\langle\tilde{\alpha}_{i_{0}-}\right|\right\}$, and

the projection $\left|\tilde{\alpha}_{i_{0}-}\right\rangle\left\langle\tilde{\alpha}_{i_{0}-}\right|$ was successful. Thus $\beta$ was actually steered into $\left|\tilde{\phi}_{i_{0}-}\right\rangle$, i.e., the chaos effect can indeed occur physically.

It is also worth further studying whether such chaos could be at least one of the origins of quantum uncertainty in measurements. We would like to leave it open for future discussions.

\section{ANOMALOUS BEHAVIOR IN INFINITE-DIMENSIONAL SYSTEMS}

When taking the $n \rightarrow \infty$ limit in the above equations, the result will be even more interesting. We shall prove below that it will lead to Conclusion 2 .

Conclusion 2: In infinite-dimensional systems, it is impossible to construct a bipartite system $\alpha \otimes \beta$, such that by the local measurement on system $\alpha$ alone, the state of system $\beta$ can be steered between the following two sets of evenly distributed states

$$
\left\{\left|\phi_{i+}\right\rangle \equiv \frac{1}{\sqrt{2}}(|0\rangle+|i\rangle), i=1, \ldots, n-1\right\}
$$

and

$$
\left\{\left|\phi_{i-}\right\rangle \equiv \frac{1}{\sqrt{2}}(|0\rangle-|i\rangle), i=1, \ldots, n-1\right\}
$$

where $n \rightarrow \infty$. 
That is, we are going to prove that for any bipartite system $\alpha \otimes \beta$, it is impossible to find two different measurements $M_{+}$and $M_{-}$, such that applying $M_{+}$on $\alpha$ will make $\beta$ collapse to one of the states in set $\left\{\left|\phi_{i+}\right\rangle\right\}$, while applying $M_{-}$on $\alpha$ instead will make $\beta$ collapse to one of the states in set $\left\{\left|\phi_{i-}\right\rangle\right\}$.

Let us start the proof by assuming that there is a bipartite system $\alpha \otimes \beta$ which can steer the state of $\beta$ to the elements of set $\left\{\left|\phi_{i+}\right\rangle\right\}$. Then there must exist a local measurement $M_{+}$, such that applying it on $\alpha$ will yield an index $i$ of state $\left|\phi_{i+}\right\rangle$ to which $\beta$ will have collapsed. Denote the eigenstates of $M_{+}$as $\left|\alpha_{i+}\right\rangle(i=0, \ldots, n-1)$, i.e.,

$$
M_{+}=\sum_{i=0}^{n-1} i\left|\alpha_{i+}\right\rangle\left\langle\alpha_{i+}\right| .
$$

Then the state of $\alpha \otimes \beta$ can surely be written in the form of Eq. (11). In brief, any system that can steer the state of its part into set $\left\{\left|\phi_{i+}\right\rangle\right\}$ will take the form of Eq. (1) as long as the basis $\left\{\left|\alpha_{i+}\right\rangle, i=0, \ldots, n-1\right\}$ is properly defined.

Now the question is whether such a system can be steered into the states in set $\left\{\left|\phi_{i-}\right\rangle\right\}$. If the answer is yes, then it means that there exists another local measurement $M_{-}$, such that applying it on $\alpha$ will yield an index $i$ of state $\left|\phi_{i-}\right\rangle$ to which $\beta$ will have collapsed. Denote the eigenstates of $M_{-}$as $\left|\alpha_{i-}\right\rangle(i=0, \ldots, n-1)$, i.e.,

$$
M_{-}=\sum_{i=0}^{n-1} i\left|\alpha_{i-}\right\rangle\left\langle\alpha_{i-}\right| .
$$

Then the same $|\Omega\rangle$ in Eq. (11) should also be able to be expanded in the basis $\left\{\left|\alpha_{i-}\right\rangle\right\}$ as

$$
|\Omega\rangle=\frac{1}{\sqrt{n-1}} \sum_{i=1}^{n-1}\left|\alpha_{i-}\right\rangle\left|\phi_{i-}\right\rangle .
$$

Let us study what is the relationship between the measurements $M_{+}$and $M_{-}$.

Since the equations in the previous section are valid for any $n$, they also apply when $n \rightarrow \infty$. So we can still obtain Results 1 and 2 that projecting system $\alpha$ into $\left|\alpha_{i+}\right\rangle$ (or $\left|\tilde{\alpha}_{i-}\right\rangle$ ) will make system $\beta$ collapse into $\left|\phi_{i+}\right\rangle$ (or $\left|\tilde{\phi}_{i-}\right\rangle$ ).

But multiplying $\left\langle\alpha_{i+}\right|$ by the right-hand side of Eq. (8), we have

$$
\left\langle\alpha_{i+} \mid \tilde{\alpha}_{i-}\right\rangle=\frac{2-n}{n} c_{-}=-\sqrt{1-\frac{4}{n+2}} .
$$

Likewise, multiplying $\left\langle\phi_{i-}\right|$ by the right-hand side of Eq. (16) gives

$$
\left\langle\phi_{i-} \mid \tilde{\phi}_{i-}\right\rangle=\sqrt{1-\frac{2 n+2}{n^{2}+2}}
$$

Therefore, in the $n \rightarrow \infty$ limit we have

$$
\left|\tilde{\alpha}_{i-}\right\rangle=-\left|\alpha_{i+}\right\rangle
$$

and

$$
\left|\tilde{\phi}_{i-}\right\rangle=\left|\phi_{i-}\right\rangle .
$$

That is, in infinite-dimensional systems, Result 2 becomes Result 2'.

Result 2': If we manage to project system $\alpha$ into $-\left|\alpha_{i+}\right\rangle$, then $\beta$ will collapse into $\left|\phi_{i-}\right\rangle$.

Now recall that we assumed that Eq. (23) also applies to this system, which implies that if we manage to project system $\alpha$ into $\left|\alpha_{i-}\right\rangle$, then $\beta$ will collapse into $\left|\phi_{i-}\right\rangle$. Comparing with Result 2' and Eq. (26), we know that

$$
\left|\alpha_{i-}\right\rangle=\left|\tilde{\alpha}_{i-}\right\rangle=-\left|\alpha_{i+}\right\rangle
$$

and therefore

$$
\left|\alpha_{i-}\right\rangle\left\langle\alpha_{i-}|=| \alpha_{i+}\right\rangle\left\langle\alpha_{i+}\right|
$$


for $i=1, \ldots, n-1$. Also, since $\sum_{i=0}^{n-1}\left|\alpha_{i \pm}\right\rangle\left\langle\alpha_{i \pm}\right|=I$ with $I$ being the identity matrix, we have

$$
\left|\alpha_{0-}\right\rangle\left\langle\alpha_{0-}\left|=I-\sum_{i=1}^{n-1}\right| \alpha_{i-}\right\rangle\left\langle\alpha_{i-}\left|=I-\sum_{i=1}^{n-1}\right| \alpha_{i+}\right\rangle\left\langle\alpha_{i+}|=| \alpha_{0+}\right\rangle\left\langle\alpha_{0+}\right| .
$$

Substituting these into Eqs. (21) and (22), we find

$$
M_{+}=M_{-} .
$$

These equations mean that if we want to collapse $\beta$ into one of the states in the set $\left\{\left|\phi_{i+}\right\rangle\right\}$ (or $\left\{\left|\phi_{i-}\right\rangle\right\}$ ), then we should measure $\alpha$ in the basis $\left\{\left|\alpha_{i+}\right\rangle, i=0, \ldots, n-1\right\}$ (or $\left\{\left|\alpha_{i-}\right\rangle=-\left|\alpha_{i+}\right\rangle, i=0, \ldots, n-1\right\}$ ). However, as the global negative sign before the state vector has no physical meaning, these two bases are actually the same. Consequently, the measurements $M_{+}$and $M_{-}$on $\alpha$ for collapsing $\beta$ to an element of the sets $\left\{\left|\phi_{i+}\right\rangle\right\}$ and $\left\{\left|\phi_{i-}\right\rangle\right\}$, respectively, are no longer two different measurements when $n \rightarrow \infty$.

Thus it is shown that for any bipartite system $\alpha \otimes \beta$ which can steer the state of $\beta$ to the elements of set $\left\{\left|\phi_{i+}\right\rangle\right\}$, if we want to steer the state of $\beta$ to the elements of set $\left\{\left|\phi_{i_{-}}\right\rangle\right\}$instead, we will find that the corresponding measurement $M_{-}$is completely indistinguishable from the measurement $M_{+}$ for steering $\beta$ to $\left\{\left|\phi_{i+}\right\rangle\right\}$. This completes the proof that it is impossible to find a bipartite system which can steer one of its part between the states $\left\{\left|\phi_{i+}\right\rangle\right\}$ and $\left\{\left|\phi_{i-}\right\rangle\right\}$ by choosing different measurements on the other part.

Some might wonder whether it is legitimate to take the $n \rightarrow \infty$ limit in our above equations and those in the Appendix. But in fact this impossibility of steering can be found even for finite $n$, as long as it is sufficiently high. This is because all physical measurement devices are subjected to the uncertainty principle, so that they cannot be manufactured and adjusted with unlimited precision. When $n$ rises to an extremely high (but still finite) value, even though Eq. (26) is not rigorously satisfied, $\left\langle\alpha_{i+} \mid \tilde{\alpha}_{i-}\right\rangle$ will become so close to -1 [as shown by Eq. (24)] that distinguishing $\left|\tilde{\alpha}_{i-}\right\rangle$ and $-\left|\alpha_{i+}\right\rangle$ will require extremely subtle adjustment on the measurement devices (such as controlling the width of the slits in interference systems, twisting the angles of the lens, etc.), which falls within the Planck scale. Such subtle adjustment is inaccessible, both theoretically and practically. Thus $\left|\tilde{\alpha}_{i-}\right\rangle$ and $-\left|\alpha_{i+}\right\rangle$ become physically indistinguishable, so that even if the measurements $M_{+}$and $M_{-}$are not strictly equal to each other, distinguishing them is still impossible. As a consequence, the local measurements on $\alpha$ are insufficient for steering $\beta$ between $\left\{\left|\phi_{i+}\right\rangle\right\}$ and $\left\{\left|\phi_{i-}\right\rangle\right\}$ even in such a finite-dimensional bipartite system.

\section{SIGNIFICANCE OF THE RESULT}

It might look trivial just to find two sets of states, and prove that it is impossible to realize the quantum steering between them. But a very important feature of our result, is that the two sets of states $\left\{\left|\phi_{i+}\right\rangle\right\}$ and $\left\{\left|\phi_{i-}\right\rangle\right\}$ also display another distinct property. As shown in the Appendix, the trace distance between the density matrices $\rho_{+}$ and $\rho_{-}$corresponding to $\left\{\left|\phi_{i+}\right\rangle\right\}$ and $\left\{\left|\phi_{i-}\right\rangle\right\}$, respectively, is $D\left(\rho_{+}, \rho_{-}\right)=1 / \sqrt{n-1}$, which drops as $n$ increases. Therefore, when $n$ is extremely high, $\left\{\left|\phi_{i+}\right\rangle\right\}$ and $\left\{\left|\phi_{i-}\right\rangle\right\}$ will become physically indistinguishable.

This property is important because in quantum steering, there is a well-known result called the Hughston-JozsaWootters (HJW) theorem [11]. It also appears under different names (e.g., the Uhlmann theorem) and presentations in literature 12 14]. A very concise summary of its conclusion can be found in Ref. [15], which goes as follows.

Let $\psi_{1}, \psi_{2}, \ldots, \psi_{m}$ and $\psi_{1}^{\prime}, \psi_{2}^{\prime}, \ldots, \psi_{m^{\prime}}^{\prime}$ be two sets of possible quantum states with associated probabilities described by an identical density matrix $\rho$. It is possible to construct a composite system $\alpha \otimes \beta$ such that $\beta$ alone has density matrix $\rho$ and such that there exists a pair of measurements $M_{\psi}$ and $M_{\psi^{\prime}}$ with the property that applying $M_{\psi}\left(M_{\psi^{\prime}}\right)$ to $\alpha$ yields an index $i$ of state $\psi_{i}\left(\psi_{i}^{\prime}\right)$ to which $\beta$ will have collapsed.

The original proofs of the HJW theorem are defined only for finite-dimensional ensembles. Later, they were generalized to the infinite-dimensional ensembles [16, 17]. But our result seems to conflict with this theorem, as we showed that for extremely high $n$, constructing such a composite system to steer between the two sets $\left\{\left|\phi_{i+}\right\rangle\right\}$ and $\left\{\left|\phi_{i-}\right\rangle\right\}$ defined in Eqs. (19) and (20) is impossible, even though their trace distance $D\left(\rho_{+}, \rho_{-}\right)$can be made arbitrarily small.

Nevertheless, after checking the existing proofs of the HJW theorem [11 14, 16, 17] carefully, this is not surprising because of the following two reasons.

(1) These proofs are valid for two ensembles with exactly the same density matrix that spans the same subspace. In contrast, in our case, the two density matrices corresponding to the sets $\left\{\left|\phi_{i+}\right\rangle\right\}$ and $\left\{\left|\phi_{i-}\right\rangle\right\}$ merely approach to each other in the $n \rightarrow \infty$ limit. For any finite $n$, the subspaces that they span are never exactly the same. The proofs of the HJW theorem were never claimed to be applied to such cases. 
(2) Those proofs merely predicted the existence of two measurements $M_{\psi}$ and $M_{\psi^{\prime}}$. They have not shown explicitly how different the two measurements are. Our above analysis clearly gives the relationship between the two measurements $M_{+}$and $M_{-}$on $\alpha$ for collapsing $\beta$ to an element of $\left\{\left|\phi_{i+}\right\rangle\right\}$ and $\left\{\left|\phi_{i-}\right\rangle\right\}$, respectively. That is, the corresponding measurement bases are $\left\{\left|\alpha_{i+}\right\rangle, i=0, \ldots, n-1\right\}$ and $\left\{\left|\alpha_{i-}\right\rangle=-\left|\alpha_{i+}\right\rangle, i=0, \ldots, n-1\right\}$ ), where the difference is merely a global negative sign which has no physical meaning. Thus we can see that although our final outcome (that steering between these two specific sets is impossible) looks different from the prediction of the HJW theorem (as summarized above), there is no theoretical conflict between the proofs of that theorem and ours.

The properties of $\left\{\left|\phi_{i+}\right\rangle\right\}$ and $\left\{\left|\phi_{i-}\right\rangle\right\}$ that we found above could make them very useful in quantum cryptography. There are many no-go theorems in this field, such as the impossibility of unconditionally secure quantum bit commitment [18, 19], and the insecurity proof of two-party quantum secure computations [20]. They are all based directly on the above-mentioned conclusion of the HJW theorem. In brief, the cheater begins these quantum cryptographic tasks using entangled states, although in the honest case he is expected to use either $\psi_{i}$ or $\psi_{i}^{\prime}$ alone, where the density matrices of the sets $\left\{\psi_{i}\right\}$ and $\left\{\psi_{i}^{\prime}\right\}$ are required to be identical (so that the other party cannot distinguish them). At the end of the process, he chooses between the two measurements $M_{\psi}$ and $M_{\psi^{\prime}}$ on his own system $\alpha$, so that system $\beta$ held by the other party will collapse either to $\psi_{i}$ or $\psi_{i}^{\prime}$ at the cheater's own choice, just as it is described in the HJW theorem. With this method, the cheater can gain extra advantage than what is allowed in the honest case, making the corresponding cryptographic protocol insecure. But our result shows that when $\psi_{i}$ and $\psi_{i}^{\prime}$ are taken as $\left|\phi_{i+}\right\rangle$ and $\left|\phi_{i-}\right\rangle$ in Eqs. (19) and (20), the two measurements $M_{\psi}$ and $M_{\psi^{\prime}}$ become indistinguishable for infinite-dimensional systems, so that the cheater no longer has the freedom to choose the state to which $\beta$ will collapse. Meanwhile, the two sets $\left\{\left|\phi_{i+}\right\rangle\right\}$ and $\left\{\left|\phi_{i-}\right\rangle\right\}$ also meet the requirement that they can be indistinguishable to the other party. Therefore, quantum protocols built upon the states $\left\{\left|\phi_{i+}\right\rangle\right\}$ and $\left\{\left|\phi_{i_{-}}\right\rangle\right\}$may eventually evade the cheating strategy in these no-go theorems. We will study such protocols in forthcoming works.

\section{SUMMARY}

We showed that when a finite-dimensional bipartite system $\alpha \otimes \beta$ is prepared in the state described in Eq. (1), it can display a chaos effect in quantum steering, such that a subtle difference in the measurement results on $\alpha$ can lead to completely orthogonal steering results on $\beta$.

For infinite-dimensional systems, we showed that it is impossible to construct a bipartite system $\alpha \otimes \beta$ which can steer the state of system $\beta$ between the two sets defined in Eqs. (19) and (20), even though the trace distance between the density matrices of the two sets can be made arbitrarily small as $n$ increases. This result could be very useful for quantum cryptography.

It is also interesting to study whether there exist other forms of states which can lead to similar anomalous behavior in steering, especially in finite-dimensional systems.

The author thanks Dr. Shuming Cheng for helpful discussions.

\section{Appendix A: The trace distance}

Let $\rho_{+}$and $\rho_{-}$denote the density matrices of the two sets of evenly distributed states $\left\{\left|\phi_{i+}\right\rangle\right\}$ and $\left\{\left|\phi_{i-}\right\rangle\right\}$ defined in Eqs. (19) and (20), respectively. Here we calculate the trace distance between $\rho_{+}$and $\rho_{-}$.

Denote

$$
\begin{aligned}
& \rho_{0 \pm i} \equiv \frac{1}{\sqrt{2}}(|0\rangle \pm|i\rangle) \frac{1}{\sqrt{2}}(\langle 0| \pm\langle i|) \\
& =\frac{1}{2}\left[\begin{array}{c}
1 \\
0 \\
\vdots \\
0 \\
\pm 1 \\
0 \\
\vdots \\
0
\end{array}\right]\left[\begin{array}{llllllll}
1 & 0 & \cdots & 0 & \pm 1 & 0 & \cdots & 0
\end{array}\right]=\left[\begin{array}{cccccccc}
\frac{1}{2} & 0 & \cdots & 0 & \pm \frac{1}{2} & 0 & \cdots & 0 \\
0 & 0 & \cdots & 0 & 0 & 0 & \cdots & 0 \\
\vdots & \vdots & \ddots & \vdots & \vdots & \vdots & \vdots & \vdots \\
0 & 0 & \cdots & 0 & 0 & 0 & \cdots & 0 \\
\pm \frac{1}{2} & 0 & \cdots & 0 & \frac{1}{2} & 0 & \cdots & 0 \\
0 & 0 & \cdots & 0 & 0 & 0 & \cdots & 0 \\
\vdots & \vdots & \vdots & \vdots & \vdots & \vdots & \ddots & \vdots \\
0 & 0 & \cdots & 0 & 0 & 0 & \cdots & 0
\end{array}\right] .
\end{aligned}
$$


Then for any finite $n$, when each state $(|0\rangle \pm|i\rangle) / \sqrt{2}(i=1,2, \ldots, n-1)$ in Eq. (19) and (20) occurs with equal probabilities $1 /(n-1)$, the corresponding density matrices are

$$
\rho_{ \pm}=\frac{1}{n-1} \sum_{i=1}^{n-1} \rho_{0 \pm i}=\left[\begin{array}{cccccccc}
\frac{1}{2} & \pm \frac{1}{2(n-1)} & \pm \frac{1}{2(n-1)} & \pm \frac{1}{2(n-1)} & \cdots & \pm \frac{1}{2(n-1)} & \pm \frac{1}{2(n-1)} \\
\pm \frac{1}{2(n-1)} & \frac{1}{2(n-1)} & 0 & 0 & \cdots & 0 & 0 \\
\pm \frac{1}{2(n-1)} & 0 & \frac{1}{2(n-1)} & 0 & \cdots & 0 & 0 \\
\pm \frac{1}{2(n-1)} & 0 & 0 & \frac{1}{2(n-1)} & \cdots & 0 & 0 \\
\vdots & \vdots & \vdots & \vdots & \ddots & \vdots & \vdots \\
\pm \frac{1}{2(n-1)} & 0 & 0 & 0 & \cdots & \frac{1}{2(n-1)} & 0 \\
\pm \frac{1}{2(n-1)} & 0 & 0 & 0 & \cdots & 0 & \frac{1}{2(n-1)}
\end{array}\right] .
$$

Therefore,

$$
\rho_{+}-\rho_{-}=\left[\begin{array}{ccccc}
0 & \frac{1}{n-1} & \frac{1}{n-1} & \cdots & \frac{1}{n-1} \\
\frac{1}{n-1} & 0 & 0 & \cdots & 0 \\
\frac{1}{n-1} & 0 & 0 & \cdots & 0 \\
\vdots & \vdots & \vdots & \ddots & \vdots \\
\frac{1}{n-1} & 0 & 0 & \cdots & 0
\end{array}\right]
$$

and we have

$$
\begin{gathered}
\left(\rho_{+}-\rho_{-}\right)^{\dagger}\left(\rho_{+}-\rho_{-}\right)=\left[\begin{array}{ccccc}
\frac{1}{n-1} & 0 & 0 & \cdots & 0 \\
0 & \frac{1}{(n-1)^{2}} & \frac{1}{(n-1)^{2}} & \cdots & \frac{1}{(n-1)^{2}} \\
0 & \frac{1}{(n-1)^{2}} & \frac{1}{(n-1)^{2}} & \cdots & \frac{1}{(n-1)^{2}} \\
\vdots & \vdots & \vdots & \ddots & \vdots \\
0 & \frac{1}{(n-1)^{2}} & \frac{1}{(n-1)^{2}} & \cdots & \frac{1}{(n-1)^{2}}
\end{array}\right], \\
\sqrt{\left(\rho_{+}-\rho_{-}\right)^{\dagger}\left(\rho_{+}-\rho_{-}\right)}=\left[\begin{array}{ccccc}
\frac{1}{(n-1)^{1 / 2}} & 0 & 0 & \cdots & 0 \\
0 & \frac{1}{(n-1)^{3 / 2}} & \frac{1}{(n-1)^{3 / 2}} & \cdots & \frac{1}{(n-1)^{3 / 2}} \\
0 & \frac{1}{(n-1)^{3 / 2}} & \frac{1}{(n-1)^{3 / 2}} & \cdots & \frac{1}{(n-1)^{3 / 2}} \\
\vdots & \vdots & \vdots & \ddots & \vdots \\
0 & \frac{1}{(n-1)^{3 / 2}} & \frac{1}{(n-1)^{3 / 2}} & \cdots & \frac{1}{(n-1)^{3 / 2}}
\end{array}\right],
\end{gathered}
$$

where $\left(\rho_{+}-\rho_{-}\right)^{\dagger}$ denotes the Hermitian conjugation of $\left(\rho_{+}-\rho_{-}\right)$. Thus the trace distance between $\rho_{+}$and $\rho_{-}$is

$$
D\left(\rho_{+}, \rho_{-}\right) \equiv \frac{1}{2} \operatorname{tr} \sqrt{\left(\rho_{+}-\rho_{-}\right)^{\dagger}\left(\rho_{+}-\rho_{-}\right)}=\frac{1}{\sqrt{n-1}} .
$$

[1] E. Schrödinger, Proc. Cambridge Philos. Soc. 31, 555 (1935). Discussion of probability relations between separated systems

[2] E. Schrödinger, Proc. Cambridge Philos. Soc. 32, 446 (1936). Probability relations between separated systems

[3] A. Einstein, B. Podolsky, and N. Rosen, Phys. Rev. 47, 777 (1935). Can quantum-mechanical description of physical reality be cosidered complete?

[4] H. M. Wiseman, S. J. Jones, and A. C. Doherty, Phys. Rev. Lett. 98, 140402 (2007). Steering, entanglement, nonlocality, and the Einstein-Podolsky-Rosen paradox

[5] C. Branciard, E. G. Cavalcanti, S. P. Walborn, V. Scarani, and H. M. Wiseman, Phys. Rev. A 85, 010301(R) (2012). One-sided device-independent quantum key distribution: Security, feasibility, and the connection with steering

[6] M. D. Reid, Phys. Rev. A 88, 062338 (2013). Signifying quantum benchmarks for qubit teleportation and secure quantum communication using Einstein-Podolsky-Rosen steering inequalities

[7] P. Skrzypczyk, M. Navascués, and D. Cavalcanti, Phys. Rev. Lett. 112, 180404 (2014). Quantifying Einstein-PodolskyRosen steering 
[8] J. Bowles, T. Vértesi, M. T. Quintino, and N. Brunner, Phys. Rev. Lett. 112, 200402 (2014). One-way Einstein-PodolskyRosen steering

[9] D. Cavalcanti and P. Skrzypczyk, Rep. Prog. Phys. 80, 024001 (2017). Quantum steering: a review with focus on semidefinite programming

[10] W. -Y. Sun, D. Wang, and L. Ye, Laser Phys. Lett. 14, 095205 (2017). How the relativistic motion affects EinsteinPodolsky-Rosen steering

[11] L. P. Hughston, R. Jozsa, and W. K. Wootters, Phys. Lett. A 183, 14 (1993). A complete classification of quantum ensembles having a given density matrix

[12] A. Uhlmann, Rep. Math. Phys. 9, 273 (1976). The "transition probability" in the state space of a *-algebra

[13] Ph. Pearle, Phys. Rev. D 33, 2240 (1986). Stochastic dynamical reduction theories and superluminal communication

[14] N. Gisin, Helvetica Physica Acta 62, 363 (1989). Stochastic quantum dynamics and relativity

[15] C. Crépeau, in Proc. Pragocrypt '96: 1st International Conference on the Theory and Applications of Cryptology (Czech Technical University Publishing House, Prague, 1996). What is going on with quantum bit commitment?

[16] H. Halvorson, J. Math. Phys. 45, 4920 (2004). Remote preparation of arbitrary ensembles and quantum bit commitment

[17] L. Wang, J. Hou, and X. Qi, J. Phys. A: Math. Theor. 47, 335304 (2014). Fidelity and entanglement fidelity for infinitedimensional quantum systems

[18] D. Mayers, Phys. Rev. Lett. 78, 3414 (1997). Unconditionally secure quantum bit commitment is impossible

[19] H. -K. Lo and H. F. Chau, Phys. Rev. Lett. 78, 3410 (1997). Is quantum bit commitment really possible?

[20] H. -K. Lo, Phys. Rev. A 56, 1154 (1997). Insecurity of quantum secure computations 